\title{
Agrobiodiversidad de papa nativa en la provincia de Tungurahua
}

\section{Agrobiodiversity of native potato in the Tungurahua province}

\author{
Edwin Leonardo Pallo Paredes ${ }^{1 *}$, Andrea Patricia Guapi Auquillas², Verónica María Mullo Paucar ${ }^{3}$ \\ ${ }^{1}$ Escuela Superior Politécnica de Chimborazo. Facultad de Recursos Naturales. Panamericana Sur km 1,5. Riobamba, Ecuador. \\ 凶 edwin.pallo@espoch.edu.ec; edwin_agro@hotmail.com. \\ https://orcid.org/0000-0003-3127-3872 \\ ${ }^{2}$ Escuela Superior Politécnica de Chimborazo. Facultad de Recursos Naturales. Panamericana Sur km 1,5. Riobamba, Ecuador. \\ gaaplibra@yahoo.es. \\ https://orcid.org/0000-0003-0711-6391 \\ ${ }^{3}$ Escuela Superior Politécnica de Chimborazo. Facultad de Recursos Naturales. Panamericana Sur km 1,5. Riobamba, Ecuador. \\ 凶verosil12@hotmail.com.
}

\begin{abstract}
Resumen
En la presente investigación se diagnosticó la diversidad nativa de la papa (Solanum spp.) en seis comunidades en la provincia de Tungurahua. La metodología empleada fue grupos focales, a partir de los cuales se llevaron a cabo 7 grupos focales (conformados por mujeres, líderes comunitarios y jóvenes), en los que participaron entre 8 y 10 personas por grupo focal. Como resultado, se encontró que en las seis comunidades en estudio se identificaron 32 variedades de papa, de las cuales 14 son variedades mejoradas introducidas y 18 son variedades nativas presentes. Las variedades continúan manteniéndose en las comunidades en estudio mediante el reciclaje de semillas. La diversidad de variedades está influenciada por las formas de conservación, los usos y el destino de la producción. El reciclaje de semillas es la forma más común de mantener la diversidad de semillas almacenadas en bolsas y pilas. El uso principal de la diversidad de papas nativas es la alimentación humana, con la cual se preparan diferentes platos típicos. La mayor parte de la producción está destinada a la comercialización en los diferentes mercados de la zona y en menor cantidad se destina a semillas, intercambio, autoconsumo y consumo animal.
\end{abstract}

Palabras clave: diversidad de papas nativas, grupos focales, conservación, usos.

\begin{abstract}
In the present research the potato (Solanum spp.) native diversity was diagnosed in six communities of the province of Tungurahua. The methodology used was focal group and seven focal groups (women, community leaders and young people) were organized, in which eight to 10 persons participated. Thirty-two potato varieties were identified in the six communities, from which 14 were introduced improved varieties and 18 local native varieties. The varieties continue to be maintained in the communities by seed recycling. The diversity of varieties is maintained by the conservation methods, the uses and the destination of the production. Seed recycling is the most common way to maintain the diversity of seeds stored in bags and piles. The main use of the diversity of native potatoes is human consumption preparing local dishes. Most of potato production is commercialized in local markets
\end{abstract}


and to a lesser extent production is used for seeds, exchange with neighbors, self-consumption at household and animal consumption.

Keywords: potato native diversity, focal group discussion, conservation, uses.

\section{Introducción}

Las papas nativas son autóctonas de la región alta de la zona Andina, resultado de un proceso de domesticación y selección iniciado hace 8.000 años. Por su importancia dentro de la dieta de los pueblos andinos la nombraron Kausay que significa sustento a la vida. Estas variedades se caracterizan porque no han sido manipuladas genéticamente por el hombre, son híbridos generados en forma natural por cruzamientos entre diferentes especies de papas (Monteros et al., 1999; Pinto \& Abad, 2017; Rodríguez, 2010).

Las poblaciones preincaicas daban usos diferentes a las papas, las colocaban crudas para sanar los huesos rotos, para prevenir el reumatismo y las comían mezcladas con otros alimentos para mejorar la digestión; se usaban también para medir el tiempo relacionándolo con el desarrollo del cultivo (Monteros et al., 2005).

Las papas nativas son especies cultivadas del género Solanum. Producen tubérculos variopintos y se cultivan bajo duras condiciones ambientales donde las variedades comerciales no pueden competir. Durante siglos estas papas han sido localmente seleccionadas por los campesinos andinos con el fin de subsistir bajo las severas condiciones ambientales de los Andes (Ritter et al., 2009). La biodiversidad ecuatoriana de la papa incluye 23 especies silvestres y tres diploides, triploides y tetraploides cultivados de Solanum tuberosum (Monteros \& Altamirano, 2018; Spooner et al., 2014).

Las papas nativas se caracterizan por tener formas exóticas (aplanadas, redondas, comprimidas, alargadas, con ojos profundos) y colores llamativos (amarillos, rojos, rosada o morada); mientras más oscura es la piel o pulpa de los tubérculos hay mayor contenido de antioxidantes; aportan también importantes cantidades de proteínas, fibra y minerales (Monteros et al., 1999).
Los antioxidantes, tales como los polifenoles y carotenoides poseen efectos favorables para la salud humana y están presentes en el tubérculo de papa (Cuesta-Subía et al., 2012).

Para Muñoz (2014), la calidad nutritiva de la papa nativa es evidente, estudios demuestran que es una fuente de carbohidratos que contiene menos calorías y grasas que el pan, las pastas o el arroz, además es considerada como la tercera fuente de fenoles después de la manzana y la naranja. Por la concentración de color, las pulpas pueden ser utilizadas como colorantes naturales y antioxidantes en la industria alimenticia. La presencia de carotenoides le proporcionan propiedades fotoprotectoras a la vez que promueven la comunicación intercelular y la agudeza mental (Muñoz, 2014).

Es necesario destacar que la conservación de estas variedades es resultado del proceso de domesticación, selección y conservación ancestral, consecuencia de la herencia de los habitantes de la zona de los Andes (Monteros et al., 2005). A decir de Diez, et al. (2013) las pequeñas parcelas han mantenido distintas variedades con características de tolerancia, resistencia y susceptibilidad a diversos agentes tanto ambientales como patogénicos como un mecanismo de seguridad alimentaria. El mismo autor indica que los papicultores mantienen las variedades de papa color no porque sepan sus propiedades como alimentos funcionales sino por curiosidad; de ahí la existencia de un número limitado de éstas.

La variabilidad o diversidad genética es el componente básico de la biodiversidad y se define como las variaciones heredables que ocurren en cada organismo, entre los individuos de una población y entre las poblaciones dentro de una especie. El resto de la biodiversidad se deriva de los procesos evolutivos que operan sobre las diferentes variaciones (Piñero et al., 2008).

La producción de papa en Ecuador se distribuye en tres zonas geográficas: norte, centro 
y sur. Las diferentes zonas agroecológicas están determinadas no por la latitud, sino por las relaciones entre clima, fisiografía y altura. La papa es la principal fuente de alimento para los pobladores de las zonas altas del país, con un consumo anual per cápita que fluctúa según las ciudades (Pumisacho \& Sherwood, 2002).

En Ecuador en la región andina es donde se han escogido y se mantienen especies nativas diversificadas (maíz, cucúrbitas, papa, tomate, oca, ají, etc.) manejadas con diferentes prácticas agroecológicas (Suquilanda, 2012). En el caso de la papa nativa se reportan 350 variedades, siendo la provincia de Tungurahua el espacio que registra un mayor número de cultivares perdidos, pues su presencia comercial en los mercados es limitada, además que el conocimiento y hábito de consumo de éstas ha disminuido de manera considerable en la población (Tene, 2018).

La diversidad genética de estos tubérculos en nuestro país y el peligro inminente de extinción, en el que se encuentra debido al bajo consumo, obliga a buscar alternativas de preservación como diseñar un banco de semillas que permita colectar, guardar y conservar semillas con el fin de evitar que se extinga la biodiversidad, este banco de semillas, permitirá beneficiar a todos los sectores involucrados, elevando los niveles de productividad del agricultor, con alimentos sanos, nutritivos y variados, donde se propenda a la conservación y se cree un mercado propio y rentable para los campesinos. Con un buen almacenamiento de las semillas, éstas pueden mantenerse viables en un número muy variable de años, desde uno hasta diez o más años dependiendo de la especie (Roselló \& Soriano, 2010).

En los últimos años el maíz amiláceo y la papa son los principales productos básicos de la dieta de los ecuatorianos, pues tienen un significado vital para los pueblos indígenas, además de ser considerados generadores de vida, éstos constituyen elementos fundamentales de identidad de los grupos ancestrales (Brack, 2000). La diversidad nativa de muchos productos agrícolas se mantiene por agricultores en comunidades rurales, y ésta no se ha perdido en su totalidad porque es valorada por el sabor y las costumbres que cada comunidad conserva (Piñero et al., 2008).

El presente trabajo tuvo como objetivo diagnosticar la diversidad de papa nativa presente en seis comunidades de la provincia de Tungurahua, durante este proceso se buscó establecer las formas a través de las cuales se conserva esta diversidad nativa de papa, así como las formas de consumo, los destinos de la producción, y las formas de abastecimiento de semilla para los siguientes ciclos productivos. En función de este propósito, se plantea la interrogante sobre qué está ocurriendo con la biodiversidad de papa nativa, es decir, su existencia o su ausencia en la provincia de Tungurahua.

\section{Metodología}

\subsection{Ubicación geográfica}

La investigación se realizó en seis comunidades de la provincia de Tungurahua: Pilco, Hipolongo, Yanayacu, Pilahuín, Quinchicoto y Huapante grande (Tabla 1).

Tabla 1. Coordenadas geográficas de las comunidades en estudio

\begin{tabular}{cccccc}
\hline Cantón & Parroquia & Comunidad & $\begin{array}{c}\text { Altura } \\
\text { (m s.n.m.) }\end{array}$ & Latitud & Longitud \\
\hline \multirow{2}{*}{ Quero } & Yanayacu & Yanayacu & 3.330 & $1^{\circ} 26^{\prime} 06.64^{\prime \prime} \mathrm{S}$ & $78^{\circ} 39^{\prime} 39.86^{\prime \prime} \mathrm{O}$ \\
& & Pilco & 2.250 & $1^{\circ} 25^{\prime} 55.43^{\prime \prime} \mathrm{S}$ & $78^{\circ} 34^{\prime} 02.22^{\prime \prime} \mathrm{O}$ \\
\multirow{2}{*}{ Ambato } & Pilahuín & Pipolongo & 3.200 & $1^{\circ} 25^{\prime} 30.54^{\prime \prime} \mathrm{S}$ & $78^{\circ} 38^{\prime} 54.51^{\prime \prime} \mathrm{O}$ \\
Mocha & Quinchicoto & Quinchicoto & 3.326 & $1^{\circ} 23^{\prime} 05.10^{\prime \prime} \mathrm{S}$ & $78^{\circ} 39^{\prime} 28.34^{\prime \prime} \mathrm{O}$ \\
Píllaro & Guapante & Huapante grande & 2.688 & $1^{\circ} 07^{\prime} 42.22^{\prime \prime} \mathrm{S}$ & $78^{\circ} 35^{\prime} 07.37^{\prime \prime} \mathrm{O}$ \\
\hline
\end{tabular}




\subsection{Actividades realizadas con los grupos focales}

Según Kitzinger (1995) un grupo focal es una entrevista grupal homogénea que utiliza la comunicación entre investigador y participantes (8 - 15 personas), con el propósito de conseguir información en un espacio de opinión para captar el sentir, pensar y vivir de los individuos, provocando autoexplicaciones para obtener datos cualitativos. Para la realización de los grupos focales (GF) se siguieron los siguientes pasos:

Selección de comunidades

Selección de población y muestra

Visita a las comunidades

Validación de la metodología

Desarrollo de los GF

\subsubsection{Selección de comunidades}

El trabajo se realizó en el primer semestre del año 2019, con el apoyo de líderes y productores de las comunidades en estudio. En trabajos realizados previamente, se identificó la existencia de comunidades en la provincia de Tungurahua que aún disponen de diversidad genética de papa, manteniendo variedades que, sin tener mayor importancia en los diferentes mercados, disponen de un alto grado de representación, sentido y significación cultural para los productores que las conservan.

Para seleccionar a las comunidades se establecieron criterios que permitieron identificar un segmento apropiado de informantes considerando aspectos obligatorios y otros deseables que deben cumplir los informantes:

I Requisitos obligatorios

i La comunidad debe ser organizada, es decir, realizan actividades en común entre los pobladores.

ii La principal actividad económica es la agricultura.

iii Poseer germoplasma nativo de variedades de papa, cultivadas frecuentemente, en la finca del productor o en fincas vecinas.

iv Disponer de interés para participar en el proyecto.
II Requisitos deseables

i Disponer de facilidades para el ingreso a la comunidad.

ii El destino de la producción no debe ser únicamente para el autoconsumo, sino que su flujo sea también a diferentes destinos, tales como mercados, intercambios, etc.

\subsubsection{Selección de población y muestra}

En la presente investigación los informantes fueron elegidos de forma intencionada, no probabilística, determinando un segmento de informantes que tengan conocimiento de la diversidad nativa, al igual que un segmento que no disponga de este conocimiento, segmentando la muestra en líderes comunitarios, mujeres dedicadas al campo, y jóvenes que habitan en las comunidades que cultivan papa nativa.

La obtención de la muestra se determinó mediante los requerimientos para formar un grupo focal, esto es, que cada grupo debía tener entre 10 y 15 personas, de los segmentos antes mencionados, con la finalidad de obtener distintas perspectivas de los participantes en las reuniones realizadas en cada comunidad.

\subsubsection{Visita a las comunidades}

Durante la obtención de información de los participantes se utilizó un formato guía, mismo que contiene de manera planificada los diferentes momentos y actividades que se van a desarrollar en el grupo focal. Para el efecto, fue necesario generar una base de datos con información de los líderes comunitarios.

\subsubsection{Validación de la metodología}

Antes de desarrollar los GF en las comunidades en estudio, se realizó la validación de la Guía del Grupo Focal, en la comunidad de Quinchicoto, para efectuar los ajustes necesarios.

\subsubsection{Desarrollo de los GF}

A partir de una serie de planificaciones previas con los líderes comunitarios, se establecie- 
ron las fechas y horarios para efectuar las reuniones con los GF en las comunidades en estudio.
La información recopilada a través de los GF se registró en la matriz que se muestra en la Tabla 2.

Tabla 2. Información registrada en los GF

\begin{tabular}{ll}
\hline \multicolumn{1}{c}{ Ítem } & \multicolumn{1}{c}{ Actividad } \\
\hline Variedades & $\begin{array}{l}\text { Se enlistaron todas las variedades nativas y mejoradas mencionadas por los participantes, que } \\
\text { conocen y que tienen presencia en la zona. }\end{array}$ \\
Ausentes & Se marcaron con una x las variedades de papa nativas que se han perdido. \\
Presentes & Se marcaron con una x las variedades de papa nativas que siembran actualmente. \\
$\begin{array}{l}\text { Obtención de la se- } \\
\text { milla }\end{array}$ & Se registraron las formas de obtener la semilla de papa para la siembra. \\
Almacenamiento & Se registraron las formas de almacenamiento que utilizan tanto para el consumo como para la semilla. \\
Flujos & $\begin{array}{l}\text { Se registraron los destinos de la producción, a fin de poder determinar qué cantidad destinan para } \\
\text { semilla, para el autoconsumo o para compartir. }\end{array}$ \\
Usos & Se registraron las formas de consumo de las variedades de papa. \\
\hline
\end{tabular}

\subsection{Análisis de la información a sistematizar}

Para determinar el estado de la diversidad nativa de papa, se realizaron diferentes análisis de la información obtenida en los grupos focales. Primero, se realizó una tabla de frecuencias para poder conocer los nombres de las variedades más mencionadas, con base en el número de grupos focales y en la experiencia de los agricultores. Luego, se desarrolló una escala arbitraria que se muestra en la Tabla 3 , para valorar la importancia de las diferentes variedades mencionadas. Para los indicadores de las formas de conservación y flujos, éstos se representaron gráficamente en porcentaje. El análisis para los diferentes usos de la diversidad nativa de papa se realizó en tablas donde consta el nombre del uso, una descripción y las variedades utilizadas. Con la ayuda del programa estadístico "R", se procedió a analizar la información mediante gráficos y distribuciones de frecuencias.

\section{Resultados y Discusión}

\subsection{Estado de la diversidad nativa de papa (Solanum sp.)}

En los grupos focales establecidos, para obtener información de papa nativa, se identificaron 32 nombres de variedades de papa, en las cuales están incluidas variedades mejoradas introducidas, variedades nativas presentes y variedades nativas ausentes; de las cuales el $44 \%$ corresponden a variedades mejoradas introducidas, el $21 \%$ a las variedades nativas presentes y el $35 \%$ corresponde a las variedades nativas ausentes (Figura 1).

Según los datos reportados, la diversidad de especies nativas es baja en función de las variedades introducidas; uno de los principales factores que afectan la presencia de la diversidad de papa es la falta de consumo, sobre todo por el desconocimiento, cambio de patrón de consumo principalmente de los jóvenes, los problemas climáticos que acarrean la presencia de plagas y enfermedades que ocasionan que las variedades se vuelvan más susceptibles. 


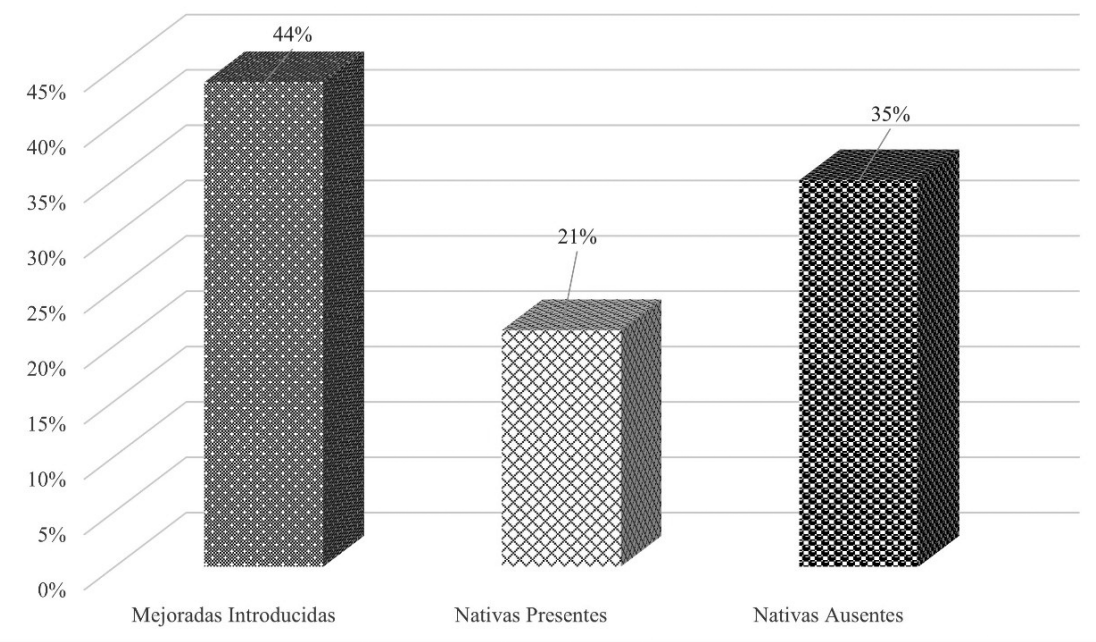

Figura 1. Diversidad de papa (Solanum spp) en seis comunidades de Tungurahua, Ecuador.

Tabla 3. Escala arbitraria para valorar estado de la diversidad de papa nativa

\begin{tabular}{cc}
\hline Rangos & Valoración \\
\hline $76-100 \%$ & Muy conocido \\
\hline $51-75 \%$ & Conocido \\
\hline $26-50 \%$ & Poco conocido \\
\hline $0-25 \%$ & Muy poco conocido \\
\hline
\end{tabular}

En la Tabla 4 se puede observar el comportamiento de las variedades reportadas en los GF en cada comunidad, si se trata de una variedad nativa presente, ausente o mejorada introducida, se puede atribuir estos cambios a las demandas actuales de la sociedad, y los cambios de hábitos del consumidor, dando como resultado que la papa nativa tenga preferencia por el agricultor y un limitado segmento de la población, los cuales reconocen las características que las diferen- cian de las demás, cabe también mencionar que se registra la existencia de 14 variedades mejoradas introducidas (Suprema, INIAP Cecilia, INIAP Esperanza, Margarita, Súper chola, Catalina, India frit, INIAP Gabriela, INIAP Fripapa, Rosita, Única, Pan, Pera y la variedad Pusas, de la cual se desconoce su origen), los agricultores cultivan variedades mejoradas ya que se obtiene una mayor productividad y ellos pueden comercializar en los diferentes mercados de la zona.

Tabla 4. Variedades de papa reportadas por las comunidades en los GF.

\begin{tabular}{|c|c|c|c|c|c|c|}
\hline \multirow[b]{2}{*}{ Variedades* } & \multicolumn{6}{|c|}{ Comunidades } \\
\hline & Pilco & Hipolongo & Yanayacu & Quinchicoto & $\begin{array}{c}\text { Huapante } \\
\text { grande }\end{array}$ & Pilahuín \\
\hline Cachito & NA & NA & NP & NP & NA & NA \\
\hline Carrizo & NA & NA & NP & NA & NA & NA \\
\hline Catalina & MI & & MI & & MI & MI \\
\hline Chaucha & $\mathrm{NP}$ & NA & NP & NP & NP & NP \\
\hline
\end{tabular}




\begin{tabular}{|c|c|c|c|c|c|c|}
\hline \multirow[b]{2}{*}{ Variedades* } & \multicolumn{6}{|c|}{ Comunidades } \\
\hline & Pilco & Hipolongo & Yanayacu & Quinchicoto & $\begin{array}{c}\text { Huapante } \\
\text { grande }\end{array}$ & Pilahuín \\
\hline Chicuila & NA & NA & NA & NP & NA & NA \\
\hline Chiwila & NA & NA & NP & NP & NA & NA \\
\hline Chola & NP & NP & NP & NP & NP & NP \\
\hline Cronada & NP & NP & NA & NP & NP & NA \\
\hline Guagra singa & NA & NA & NA & NP & NA & NP \\
\hline Huacalá & NA & NA & NP & NA & NA & NA \\
\hline INIAP Cecilia & MI & MI & & MI & & MI \\
\hline INIAP Esperanza & MI & MI & MI & & MI & MI \\
\hline INIAP Fripapa & MI & MI & & MI & MI & \\
\hline INIAP Gabriela & MI & MI & & & MI & \\
\hline India fri & MI & & MI & MI & MI & MI \\
\hline Jardinera & NA & NA & NA & NP & NA & NP \\
\hline Leona & NP & NA & NP & NP & NP & NP \\
\hline Lina & NA & NA & NA & NP & NA & NA \\
\hline Margarita & MI & MI & & & MI & MI \\
\hline Moronga & NP & NA & NA & NA & NA & NA \\
\hline Naranja & NA & NA & NA & NP & NA & NP \\
\hline Pan & MI & & MI & & MI & MI \\
\hline Pera & MI & MI & & MI & MI & MI \\
\hline Pusas & MI & MI & MI & MI & MI & MI \\
\hline Rosita & MI & MI & & MI & & MI \\
\hline Rubí & NA & NA & NA & NA & NA & NP \\
\hline Santa Rosa amarillo & NA & NA & NA & NP & NA & NA \\
\hline Santa Rosa colorado & NA & NA & NA & NP & NA & NA \\
\hline Súper chola & MI & MI & MI & MI & MI & MI \\
\hline Suprema & MI & MI & MI & MI & MI & MI \\
\hline Única & MI & MI & MI & MI & MI & MI \\
\hline Uvilla & NP & NP & NA & NA & NA & NP \\
\hline
\end{tabular}

* Mejoradas introducidas $=$ MI, Nativas Presentes $=N P$, Nativas Ausentes $=N A$

En la Figura 2 se presenta la relación positiva y negativa que tienen las variedades en estudio con las comunidades, a partir de la cual se identifica que la parroquia Quinchicoto dispone de la mayor presencia de variedades NP; además, que la parroquia Pilco es donde existe la mayor presencia de variedades MI, en tanto que la comunidad Hipolongo posee una mayor pérdida de la diversidad de papa nativa, debido a que de las 18 variedades mencionadas por los agricultores solo se conservan 3 de ellas. 


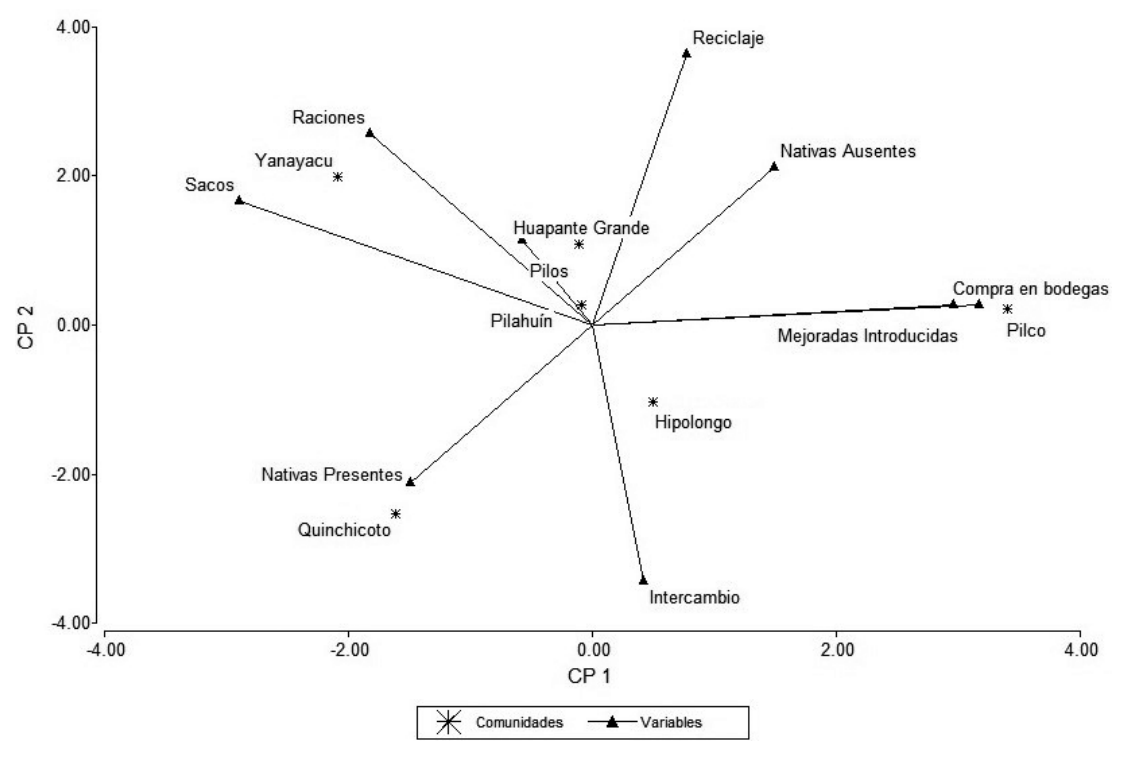

Figura 2. Análisis de componentes principales entre las comunidades de Tungurahua y las variables en estudio de papa (Solanum spp.)

A partir de los segmentos que conformaron cada GF, la Figura 3 detalla que son las mujeres quienes reconocen más diversidades de papa, identificando 7 variedades nativas presentes, 11 variedades nativas ausentes y 14 variedades mejoradas introducidas, mientras que los líderes identificaron 10 variedades nativas presentes, 8 variedades nativas ausentes y 10 variedades mejoradas introducidas.

Un $90 \%$ de los agricultores participantes en los GF de las diferentes comunidades mencionan que los jóvenes desconocen sobre la diversidad de semillas debido a que muchos de ellos han emigrado a la ciudad en busca de trabajo para poder ayudar a sus padres, o para poder estudiar; así también, indican que es más común entre los jóvenes la crianza de animales porcinos o bovinos que la actividad agrícola. Para Montero \& Altamirano (2018), las personas mayores mantienen las variedades locales, y la nueva generación demostró una falta de interés en el cultivo de variedades locales de papa. Asimismo, las mujeres poseen un gran conocimiento sobre las variedades nativas de las diferentes comunidades, debido a que ellas son las encargadas de las cosechas y de la siembra. Ellas conocen qué variedad sembrar en función de los sabores, la cantidad de producción, el tamaño de las variedades; además, disponen de conocimientos para escoger las variedades que producen los tubérculos más grandes ya que éstas les ayudan en la cocina.

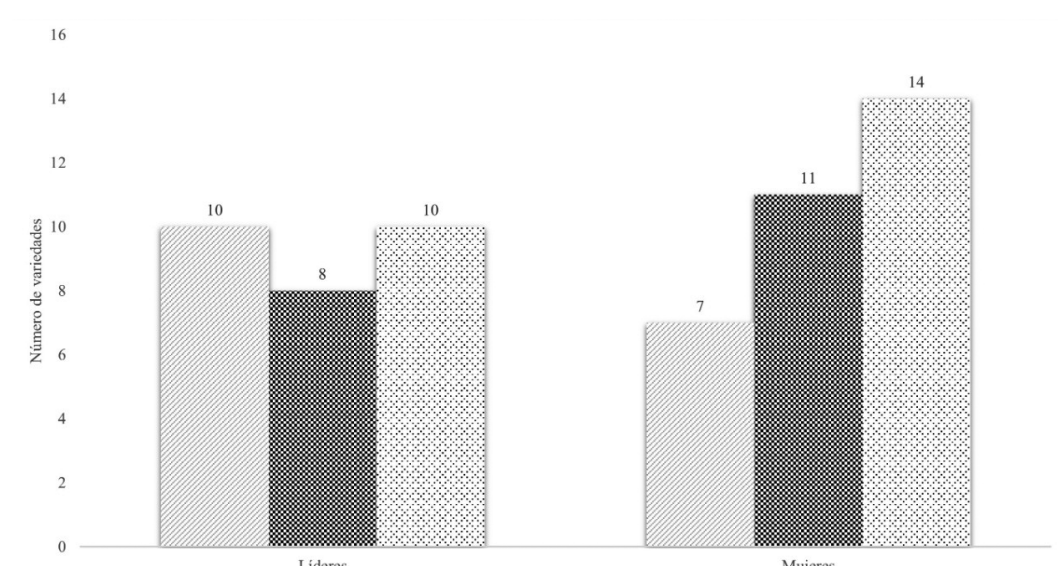

Figura 3. Información presentada por segmento de informantes en cuanto a diversidad de papa (Solanum spp) en Tungurahua Ecuador. 
Bustillos Siñani et al. (2018), manifiestan que la producción de papa nativa enfrenta factores adversos como la disponibilidad y cantidad de semilla de variedades nativas que tienen rendimientos menores, sumada a la presencia de plagas y enfermedades, helada, granizada y sequía, que provocan la disminución de la producción de papa nativa, con riesgo de desaparecer.

\subsection{Obtención y usos de la diversidad nativa de papa (Solanum spp.)}

\subsubsection{Obtención de la diversidad de papa nativa}

En la Figura 4 se reporta que el $78 \%$ de los agricultores de las seis comunidades realizan reciclaje de semillas para su siguiente ciclo de siembra; mientras que las formas de obtención con menor porcentaje corresponden a prácticas características de ciertas comunidades, con lo cual el $12 \%$ efectúa intercambio, práctica que no se realiza en las comunidades de Yanayacu y Pilahuín, debido a que los trabajadores prefieren remuneraciones económicas, antes que recibir parte de la producción por su trabajo; el $4 \%$ utiliza las raciones, la misma que es una actividad ancestral que consiste en prestar la mano para la cosecha y a cambio de ello reciben una ración de papa por su labor, acción que únicamente se desarrolla en la comunidad de Yanayacu; seguido del $4 \%$ que obtiene las semillas por chalas ${ }^{1}$ y el $2 \%$ compra las semillas en bodegas, siendo estas dos últimas formas de obtención una práctica efectuada solamente en la comunidad de Pilco. Todas estas relaciones pueden apreciarse de forma gráfica en la Figura 2, que detalla las relaciones positivas y negativas entre las comunidades y las formas de obtención de la semilla.

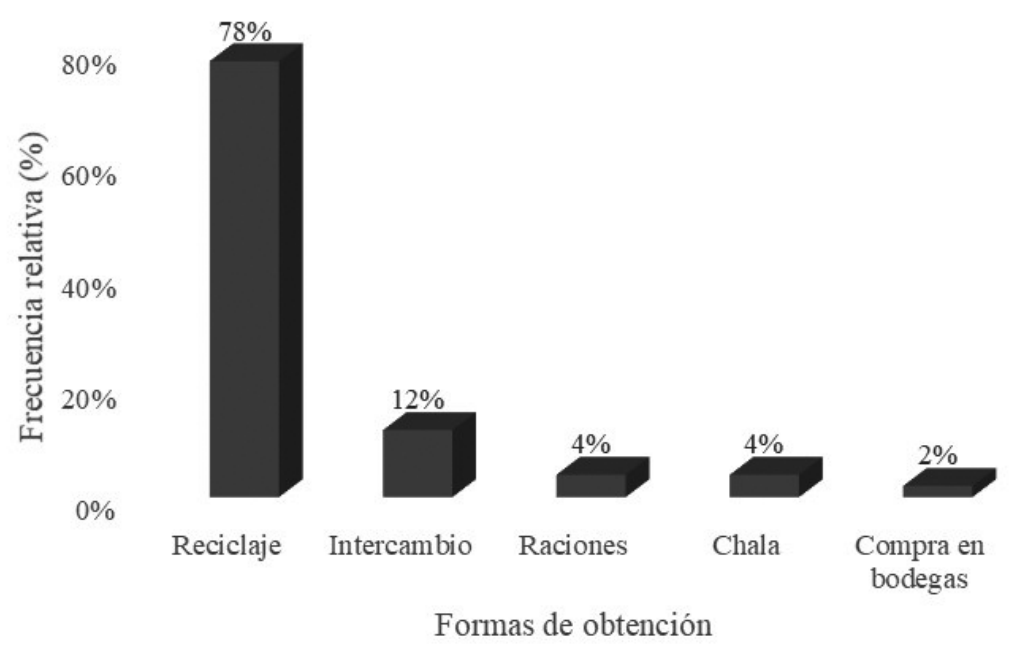

Figura 4. Formas de obtención de semillas de papa nativa en seis comunidades de Tungurahua Ecuador.

A partir de las actividades que realizan los agricultores de las diferentes comunidades en estudio para la obtención de semillas, también se puede mostrar el ingreso y salida de semillas de los cultivos lo que se da a través del reciclaje de semilla, intercambio, raciones (lo realizan la mayoría de ellos), chalas ${ }^{1}$ (práctica que viene realizándose desde hace mucho tiempo) y, por último, la compra en bodegas. En el estudio realizado por Quispe Ecos (2013) la mayor parte de los agricultores en el Perú también utilizan su propia semilla procedente de la campaña anterior, y solo el $6.3 \%$ (Huayana) y 4. 5\% (Pomacocha), realizan la compra de la semilla a otros agricultores de la zona. No realizan la compra en la ciudad de 
Andahuaylas porque carecen de recursos que les permita costear el precio y traslado hacia sus propias unidades productivas.

\subsubsection{Métodos de almacenamiento de semillas}

Así también, en la Figura 5 se observa que los agricultores de las 6 comunidades de Tungurahua almacenan la semilla en sacos y pilos; destacándose que esta práctica es más recurrente en las comunidades de Yanayacu y Huanpante Grande; mientras que el almacenamiento en pilos ${ }^{2}$ es una práctica que no se realiza en la comunidad Pilahuín.
Con respecto al porcentaje de uso de las formas más comunes de almacenamiento, se puede apreciar en la Figura 5 que el $70 \%$ del almacenamiento de la semilla se efectúa en sacos que posteriormente son guardados en bodegas que estén libres de humedad, o en los rincones de los patios (Figura 6); en tanto que el $30 \%$ almacena en pilos (Figura 7), que al igual que los sacos son colocados en bodegas o a la intemperie. Es necesario destacar que para almacenar la semilla los agricultores escogen la papa de tamaño medio que esté libre de enfermedades o plagas, ellos las almacenan en sus casas ya que tienen sus cultivos cerca de ellas.

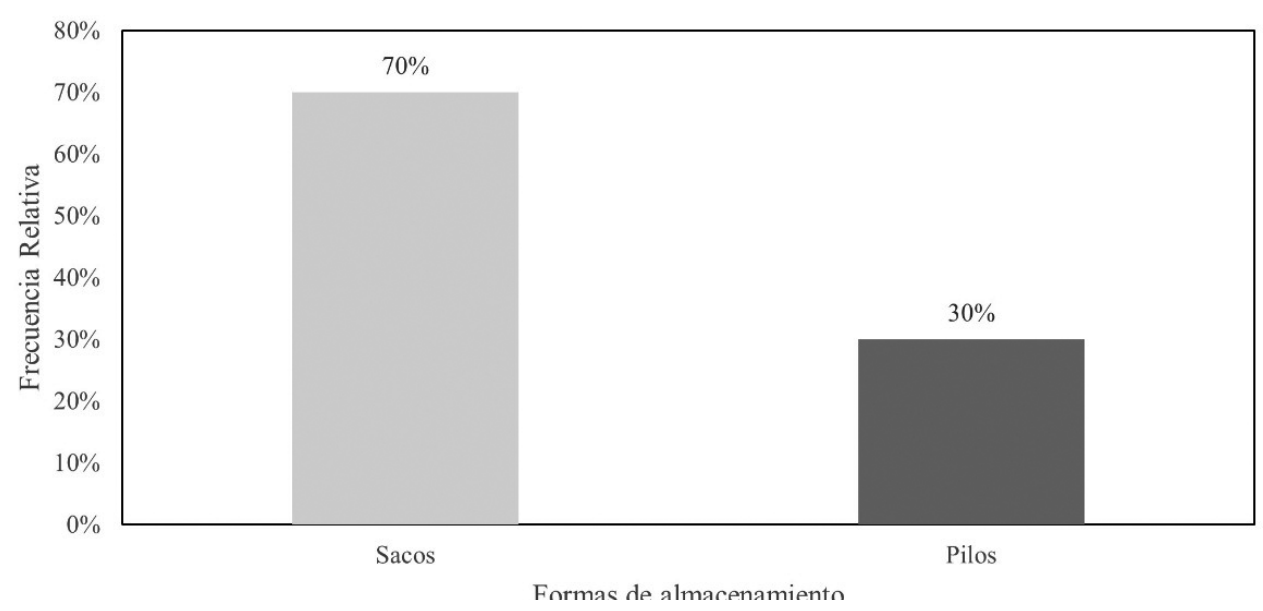

Figura 5. Formas de almacenamiento de la diversidad de papa nativa, en seis comunidades de Tungurahua Ecuador.

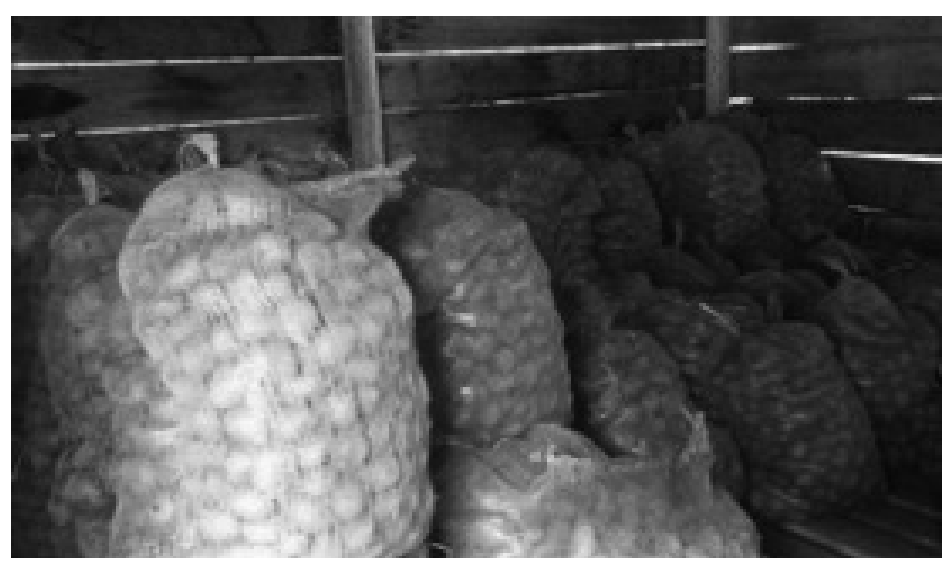

Figura 6. Almacenamiento de papa en sacos, en seis comunidades de la provincia de Tungurahua. 


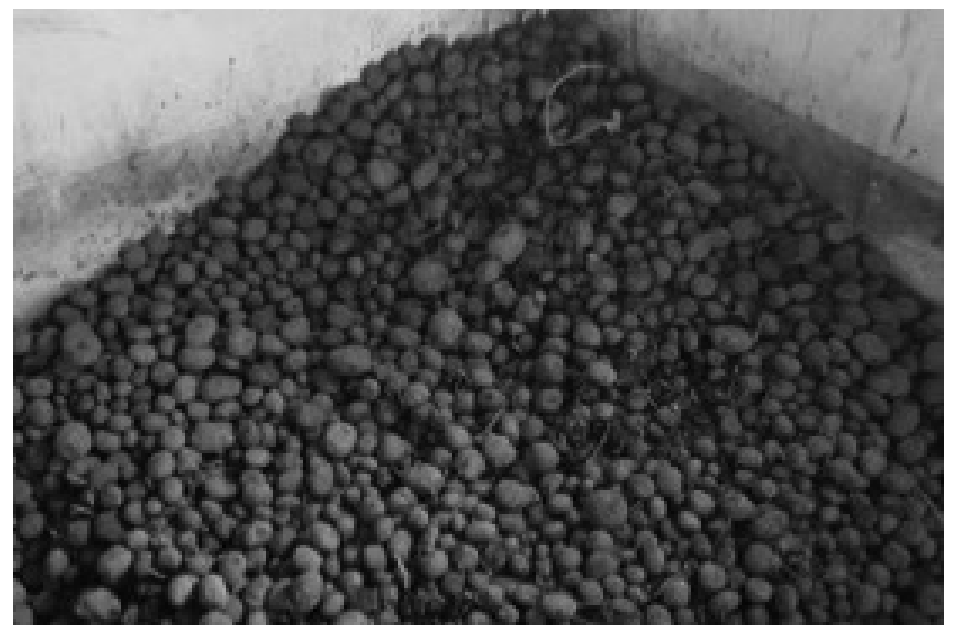

Figura 7. Almacenamiento de papa en pilos, en seis comunidades de la provincia de Tungurahua.

\subsubsection{Criterios de selección de la semilla}

En la Tabla 5 se observa que para la selección de semilla se toman en cuenta varios criterios, entre ellos están el tamaño, la sanidad y el color, todas estas cualidades ayudan al agricultor a escoger una buena semilla para el próximo cultivo y así asegurar una producción de buena calidad y cantidad.

Tabla 5. Criterios de selección para semillas de papa (Solanum spp.) en seis comunidades de Tungurahua Ecuador

\begin{tabular}{ll}
\hline \multicolumn{1}{c}{ Criterios de selección } & \multicolumn{1}{c}{ Descripción } \\
\hline Tamaño & $\begin{array}{l}\text { Las semillas de tamaño medio que tengan entre } 30 \text { y } 60 \text { gramos, son escogidas por los agriculto- } \\
\text { res para la siembra. }\end{array}$ \\
Sanidad & $\begin{array}{l}\text { Los agricultores se fijan que las semillas estén libres de cualquier enfermedad y ataque de insectos } \\
\text { o plagas, puesto que esto asegura una buena germinación. }\end{array}$ \\
Madurez & $\begin{array}{l}\text { Para utilizar los tubérculos como semilla no deben estar pelones, es decir, no se desprende con } \\
\text { facilidad su cascara. }\end{array}$ \\
\hline
\end{tabular}

\subsubsection{Usos}

Seguido, en la Tabla 6 se muestra cómo los agricultores usan todas las variedades de papa en varios platos típicos, dichas variedades de papa se caracterizan por poseer un sabor único y generar efecto notable en la preparación y presentación, por esta razón se conserva la diversidad de variedades nativas de papa en sus parcelas. En el estudio realizado por Monteros et al. (2005) las características para el uso de las variedades nativas son su buen sabor, su textura, que va de lige- ramente arenosa a arenosa; además del tiempo de cocción que va de 10 a 25 minutos a diferencia de las variedades mejoradas que se demoran $30 \mathrm{mi}-$ nutos. Las principales preparaciones en el estudio de Monteros et al. (1999) coinciden en locros, con cáscara o papas secas, fritas. Mientras que en el estudio de Cóndor (2018), presenta una gama de platos gourmet que se pueden preparar con la papa, como son: crepe de papa, croquetas, pastel de papa, etc., a diferencia de nuestro estudio que se presentan platos, tales como las tortillas, papas con cuy y papas con cuero. 
Tabla 6. Principal uso de la diversidad de papa (Solanum spp.) en seis comunidades de Tungurahua

\begin{tabular}{llc}
\hline Usos de las papas & \multicolumn{1}{c}{ Descripción } & Variedad \\
\hline Papas con cuero & $\begin{array}{l}\text { Las papas con cuero y queso, es un plato que tiene gran demanda al llegar a la provincia de } \\
\text { Tungurahua. }\end{array}$ & Todas \\
\hline Papas con cuy & Las papas con cuy y las papas con cuero son los platos más deleitados por los turistas. & Todas \\
\hline Tortillas & $\begin{array}{l}\text { Es un plato tradicional de Ambato, hecho de tortillas de papa acompañada con chorizo, } \\
\text { ensalada de remolacha y huevo frito. }\end{array}$ & Todas \\
\hline Locro & $\begin{array}{l}\text { Se sirve con queso, éste se constituye en una sopa tradicional del Ecuador, que se sirve con } \\
\text { aguacate y ají. }\end{array}$ & Todas \\
\hline Papas fritas & $\begin{array}{l}\text { Se lo prepara cortando en forma de bastones el tubérculo, los cuales se fríen en aceite y se } \\
\text { sirve con cualquier tipo de acompañamiento. }\end{array}$ & Unica \\
\hline $\begin{array}{l}\text { Chicharrón con } \\
\text { papas }\end{array}$ & Se sirve con mote y ensalada, además de ser considerado un plato tradicional de Tungurahua. & Todas \\
\hline Papas secas & $\begin{array}{l}\text { También llamada papas enteras, estas se preparan con toda la cáscara y se sirven } \\
\text { acompañadas con ají. }\end{array}$ & Todas \\
\hline
\end{tabular}

\subsection{Destino de la diversidad nativa de papa (Solanum spp.)}

Como se observa en la Figura 8, los agricultores tienen varios usos para las variedades de papa, se reporta que del $100 \%$ de la cosecha, se comercializa un $65 \%$, puesto que con ello generan ingresos económicos para el sustento de la familia; luego un $25 \%$ se destina para la semilla, un $10 \%$ para el autoconsumo y un $5 \%$ para el consumo animal, cabe recalcar que todos los desechos que se generan en la cosecha o del consumo son reciclados para la preparación de composteras, o se destinan para los animales o colocan en el propio cultivo.

De esta manera se establece que los agricultores de las comunidades de la provincia de Tungurahua se trasladan a los diferentes mercados cercanos de la comunidad para efectuar la comercialización. Lo que sobra de la comercialización lo utilizan para autoconsumo (platos típicos de la parroquia, papas a la francesa, locros) y también para consumo de animales.

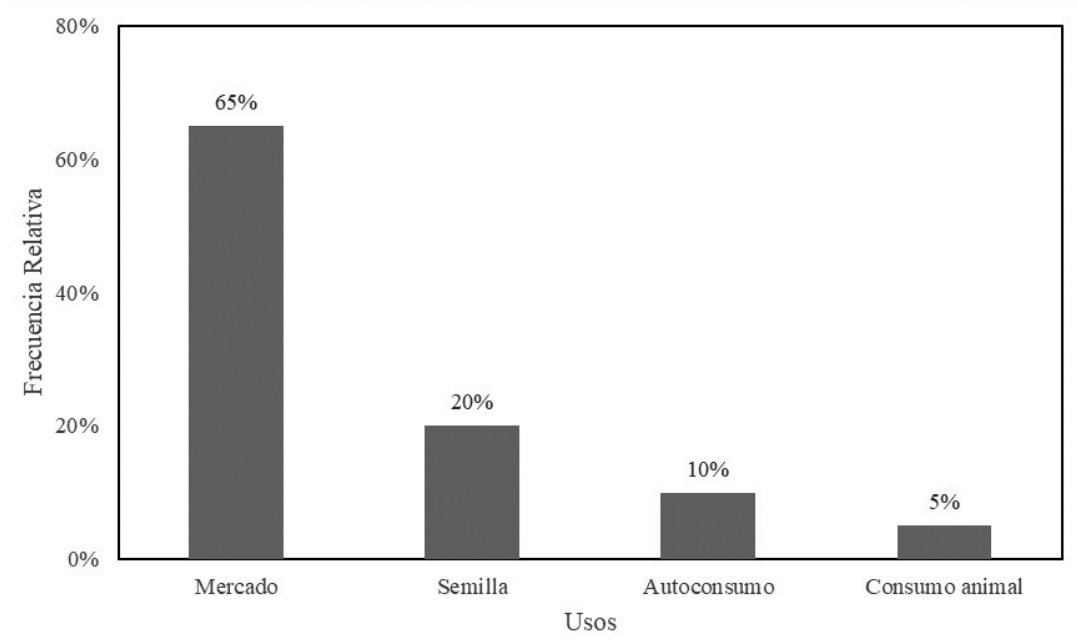

Figura 8. Destino de la producción de variedades de papa nativa. 


\section{Conclusiones}

Se identificaron 32 variedades de papa, de las cuales 14 son variedades mejoradas introducidas, y 18 son variedades nativas presentes. La forma más común utilizada por los agricultores para obtener semillas de papa es el reciclaje con el $78 \%$, en menor porcentaje está la compra en bodegas con el $2 \%$, y entre las formas intermedias están el intercambio, raciones y la chala con $12 \%, 4 \%$ y $4 \%$, respectivamente. Las formas más frecuentes de almacenar las semillas de papa son en sacos y en pilos. Se identificaron 7 formas diferentes de preparación en las cuales se utilizan todas las variedades de papa para la elaboración de platos típicos de la zona. El destino de la producción corresponde en un $65 \%$ para los mercados, el resto de la producción es utilizado para autoconsumo, semilla y para alimentación animal.

Finalmente, se puede establecer que los hábitos de consumo modernos, el desconocimiento de la población sobre las bondades nutricionales, y el valor cultural de las papas nativas, afectan en gran medida la demanda de producción de este tipo de variedades. Irónicamente, la introducción de variedades mejoradas también contribuye en la desaparición de variedades nativas.

\section{Referencias}

Brack, A. (2000). Diversidad biológica y mercados en Perú: el problema agrario en debate. Perú: SEPIA VIII.

Bustillos Siñani, L., Rojas Pardo, A., Fernández Chávez, C., \& Aparicio Porres, J. J. (2018). Identificación de variedades de papa nativa (Solanum sp.) producidas en tres comunidades del municipio de Tiahuanaco. Revista de Investigación e Innovación Agropecuaria y de Recursos Naturales, 5(2), 117-124.

Cóndor, V. (2008). Identificación de papas producidas y cultivadas en la provincia de Tungurahua: sus características y sugerencia de usos en la cocina diaria Universidad San Francisco de Quito.
Cuesta-Subía, X., Monteros-Altamirano, A., Van Den Berg, R., Visser, R. G. F., \& Vosman, B. (2012). Tuber quality characteristics of Ecuadorian potato landraces and farmer preferences. Revista Latinoamericana de la Papa, 17(1), 126-151. https://doi. org/10.37066/ralap.v17i1.191

Diez, C., López, S., Zavaleta C., Rodríguez M., \& Huayanay J. (2013). Cuantificación de los principios anticancerígenos de la variedad de papa nativa de pulpa de color de la región la libertad con el fin de procesamiento industrial. Pueblo Continente, 24(2), 425-429.

Kitzinger, J. (1995). Qualitative Research: Introducing focus groups. BMJ, 311, 299-302. https://doi.org/10.1136/bmj.311.7000.299

Monteros, C., \& Altamirano, A. (2018). Onfarm conservation of potato landraces in Ecuador. Agronomía Colombiana, 36(3) 198-200. https://doi.org/10.15446/agron. colomb.v36n3.66640

Monteros, C., Cuesta, X., Jiménez, J., \& López, G. (2005). Papas nativas en el Ecuador: Estudios cualitativos sobre oferta y demanda. Ecuador: INIAP, Estación Experimental Santa Catalina, Programa Nacional de Raíces y Tubérculos-Papa/CIP.

Monteros, C., Jiménez, J., Gavilanes, M. I., \& Reinoso, I. A. (1999). Papas nativas ecuatorianas: Redescubriendo un tesoro escondido. Ecuador: INIAP, Estación Experimental Santa Catalina, Programa Nacional de Raíces y Tubérculos Rubro-Papa.

Muñoz, M. (2014). Composición y aportes nutricionales de la papa. Revista Agrícola, 14(137), 36-37.

Pinto, M., \& Abad, A. (2017). Valor cultural del maíz y tecnologías ancestrales en la parroquia Cayambe de Ecuador. Revista Chakiñán de Ciencias Sociales y Humanidades, (2), 47-60. https://doi.org/10.37135/ chk.002.02.05 
Piñero, D., Caballero-Mellado, J., Cabrera-Toledo, D., Canteros, C., Casas, A., Castañeda, A., Castillo, A., Cerritos, R., Chassin-Noria, O., Colunga-García, N., Delgado, P., Díaz-Jaimes, P., Eguiarte L., Escalante, A., Espinoza, B., Fleury, A., Flores, S., Fragoso, G., González-Astorga, J., Islas, V., Martínez, E., Martínez, F., Martínez-Castillo, J., Mastretta, A., Medellín, R., Medrano-González, L., Molina-Freaner, F., Morales, B., Murguía, A., Vega, A., Payró, E., Reyes-Montes, M., Robles, M., Rodríguez-Arellanes, G., Rojas, L., Romero-Martínez, R., Sahaza-Cardona, J., Salas, R., Sciutto, E., Scott, C., Schramm, Y., Silva, C., Souza, V., Taylor, M., Urbán, J., Uribe-Alcócer, M., Vázquez, M., Vázquez-Domínguez, E., Vovides, A., Wegier, A., Zaldívar, A., \& Zúñiga, G. (2008). La diversidad genética como instrumento de la conservación y el aprovechamiento de la biodiversidad: estudios en especies mexicanas. En Capital natural de México, vol. I: Conocimiento actual de la biodiversidad (pp. 437-494). México: CONABIO.

Pumisacho, M., \& Sherwood, S. (Eds.) (2002). El cultivo de papa en el Ecuador. Ecuador: INIAP, Estación Experimental Santa Catalina/CIP.

Quispe Ecos, N. (2013). Análisis de la cadena de valor de la papa nativa en los distritos de Huayana y Pomacocha - Provincia de Andahuaylas - Apurímac. Pontificia Universidad Católica del Perú.
Ritter, E. Ruiz de Galarreta, J., Barandalla, L., López, R., Huarte, M., Capezzio, S., Cuesta, X., Rivadeneira, J., Vilaró, F., Gabriel, J., Scurrah, M., Canto, R., Amoros, W., Forbes, A., Bonierbale, M. (2009). Papas nativas: un cultivo con potencial de alto valor añadido para la agricultura sostenible. Revista Latinoamericana de la Papa, 15(1), 58-60. https://doi.org/10.37066/ralap.v15i1.154

Rodríguez, L. E. (2010). Origen y evolución de la papa cultivada. Una revisión. Agronomía Colombiana, 28(1), 9-17.

Roselló, J., \& Soriano, J. J. (2010). Cómo obtener tus propias semillas: Manual para agricultores ecológicos. 2. ${ }^{\text {a }}$ ed. España: Junta de Andalucía, Consejería de Agricultura y Pesca.

Spooner, D.M., Ghislain, M., Simon, R., Jansky, S. H., \& Gavrilenko, T. (2014). Systematics, Diversity, Genetics, and Evolution of Wild and Cultivated Potatoes. The Botanical Review, 80, 283-383. https://doi. org/10.1007/s12229-014-9146-y

Suquilanda, V. (2012). Producción orgánica de cultivos andinos. Ecuador: Organización de las Naciones Unidas para la Alimentación y la Agricultura (FAO).

Tene, V. (2018). Diagnóstico de la agrobiodiversidad nativa de papa (Solanum spp.) y maíz (Zea mays l.) en 5 comunidades de la provincia de Chimborazo. Escuela Superior Politécnica de Chimborazo. 